\title{
Article \\ Electron Impact Ionization of Metastable States of Diatomic Molecules
}

\author{
Annarita Laricchiuta ${ }^{1, *(\mathbb{D})}$, Roberto Celiberto ${ }^{1,2}$ (D) and Gianpiero Colonna ${ }^{1}$ (D) \\ 1 CNR ISTP (Istituto per la Scienza e Tecnologia dei Plasmi) Bari Section, 70126 Bari, Italy; \\ roberto.celiberto@poliba.it (R.C.); gianpiero.colonna@cnr.it (G.C.) \\ 2 Dipartimento di Ingegneria Civile, Ambientale, del Territorio, Edile e di Chimica (DICATECh), Politecnico di \\ Bari, 70125 Bari, Italy \\ * Correspondence: annarita.laricchiuta@cnr.it
}

check for

updates

Citation: Laricchiuta, A.; Celiberto,

R.; Colonna, G. Electron Impact

Ionization of Metastable States of

Diatomic Molecules. Atoms 2022, 10,

2. https://doi.org/10.3390/

atoms 10010002

Academic Editors: Grzegorz Piotr

Karwasz and Emmanouil P. Benis

Received: 25 October 2021

Accepted: 20 December 2021

Published: 22 December 2021

Publisher's Note: MDPI stays neutral with regard to jurisdictional claims in published maps and institutional affiliations.

Copyright: (c) 2021 by the authors. Licensee MDPI, Basel, Switzerland This article is an open access article distributed under the terms and conditions of the Creative Commons Attribution (CC BY) license (https:// creativecommons.org/licenses/by/ $4.0 /)$

\begin{abstract}
The Binary-Encounter Bethe approach was applied to the estimation of total ionization induced by electron impact in metastable states of diatomic molecules. The cross sections recently obtained for $\mathrm{N}_{2}$ and $\mathrm{CO}$ are reviewed and the new results for $\mathrm{H}_{2}$ are presented, discussing their reliability through the comparison with other theoretical methods.
\end{abstract}

Keywords: metastable states; electron-impact ionization; BEB approach; elementary processes in plasmas

\section{Introduction}

The kinetics of nonequilibrium, low-temperature plasmas is driven by the presence of radicals and excited species that can be regarded as reactivity enhancers, activating channels otherwise inaccessible and modifying the route to products. Mechanisms activated by excited species can significantly affect the efficiency of plasma technologies impacting different fields of applications, i.e., $\mathrm{CO}_{2}$ plasma reduction for environment [1], plasmaassisted combustion [2], plasma medicine, and agriculture [3,4].

The assessed theoretical framework for the description of transient and stationary conditions of such plasmas is the state-to-state approach $[5,6]$, where the quantum states of chemical species are treated independently in the master equations for the time evolution and characterized dynamically with state-specific cross sections and rate coefficients. The chemistry is coupled to the kinetics of free electrons and the internal and electron energy distributions are mutually affected. In this complex scenario, the metastable states, due to their considerably longer lifetimes with respect to radiating excited states, can play a role, acting also as a energy reservoir in the post-discharge relaxation phase and thus sustaining the plasma through the secondary collisions. This is the case of $\mathrm{N}_{2}\left(A^{3} \Sigma_{u}^{+}\right)$state in the nitrogen afterglow $[7,8]$ and also in high-enthalpy hypersonic flows [9], of $\mathrm{CO}\left(a^{3} \Pi\right)$ in $\mathrm{CO}_{2}$ discharges [10] and the odd oxygen, i.e., $\mathrm{O}_{2}\left(a^{1} \Delta_{g}\right), \mathrm{O}^{1} S$ excited states, key in the control of ignition delay time in combustion [11]. The metastable $c^{3} \Pi_{u}$ and quasi-metastable $a^{3} \Sigma_{g}^{+}$ states of $\mathrm{H}_{2}$ are relevant to the collisional radiative models for the simulation of negative ion sources for fusion [12].

Despite efforts to compile complete databases for state-resolved cross sections [13], the knowledge of data for electron scattering processes of metastables is still very scarce, thus requiring novel efforts of the quantum chemistry community. From the experimental point of view it poses difficulties entailing the preparation of the molecule in the excited state.

Focussing on the ionization process, the role of vibrational excitation of the molecular target in enhancing the ionization was investigated in the framework of classical approaches, i.e., the Gryzinski [14-19] and the universal function method [20-22], finding in general a small dependence on the vibrational quantum number. On the contrary a significant impact in the chemistry is expected for processes initiated from metastable excited states, characterized by a considerable reduction of the ionization threshold. The total ionization 
cross section of $\mathrm{N}_{2}$ metastable was obtained within the formalism of Complex Scattering Potential [23] and also partial ionization cross sections for $\mathrm{N}_{2}\left(A^{3} \Sigma_{u}^{+}\right)$and $\mathrm{O}_{2}\left(a^{1} \Delta_{g}\right)$ and $\mathrm{O}_{2}\left(b^{1} \Sigma_{g}^{+}\right)$to specific final molecular ion states were estimated in Refs. [20-22]. The $\mathrm{H}_{2}$ ionization from metastable and excited states was comprehensively investigated with the Gryzinski approach [14] and with the quantum convergent close-coupling calculations (MCCC) [24], demonstrating the predictive character of the classical approach that well reproduces the most accurate theoretical results.

In this paper, the total ionization cross sections for metastable states of diatomic molecules derived in the framework of the Binary-Encounter Bethe (BEB) approach developed by Kim\&Rudd [25,26] are discussed. The method, attractively combining simplicity and accuracy, was successfully and extensively applied to many atomic and molecular systems in the ground state, including fusion-relevant species containing heavy elements as beryllium/tungsten oxides, hydrides and nitrides [27], and also to the ionization of low-lying excited states of carbon, nitrogen and oxygen atoms [28]. Here, the results recently obtained for to the metastable states of $\mathrm{N}_{2}^{\star}$ and $\mathrm{CO}^{\star}$ [29] are reviewed and new results for $\mathrm{H}_{2}$ molecule are presented, discussing the comparison with other theoretical methods.

\section{BEB Approach}

The BEB approach $[25,26,30]$ is a derivation of the binary-encounter dipole model for electron-impact ionization of atoms and molecules, allowing the cross section estimation in those cases where the continuum dipole oscillator strength is not available.

The total ionization cross section is expressed as the sum of contributions from the electron shells

$$
\sigma_{\mathrm{BEB}}^{i o n}(E)=\sum_{i} \frac{4 \pi a_{0}^{2} N R y^{2}}{B^{2}(t+u+1)}\left[\frac{\ln t}{2}\left(1-\frac{1}{t^{2}}\right)+\left(1-\frac{1}{t}-\frac{\ln t}{t+1}\right)\right]
$$

with $R y$ the Rydberg constant, $B$ the electron binding energy in the $i$-th orbital participating in the ionization process, $N$ its occupation number. $t=E / B$ and $u=U / B, U=\left\langle\mathbf{p}^{2} / 2 m\right\rangle$ being the average kinetic energy in the orbital.

Equation (1) is obtained assuming for the the continuum dipole oscillator strength, $\frac{d f(w)}{d w}$, an analytical inverse power form

$$
\frac{d f(w)}{d w}=\frac{N}{(w+1)^{2}}
$$

where $w=W / B$, with $W=E-B$ the energy of the ejected electron.

\section{Results and Discussion}

The BEB cross sections requires the estimation of orbital parameters entering Equation (1). Electronic structure calculations were performed with the GAMESS package [31,32].

In Ref. [29] ab initio unrestricted Hartree Fock (UHF) calculations were performed, with the aug-cc-pVTZ basis set, in the $D 2 h$ symmetry point group at the equilibrium geometry of the metastable $\mathrm{N}_{2}\left(A^{3} \Sigma_{u}^{+}\right)\left(R_{e q}=1.2866 \AA\right)$. The dominant configuration at $R_{e q}$ is $\left(1 \sigma_{g}^{2} 1 \sigma_{u}^{2} 2 \sigma_{g}^{2} 2 \sigma_{u}^{2} 1 \pi_{u}^{3} 3 \sigma_{g}^{2} 1 \pi_{g}^{1}\right)$. Following the procedure recommended in Ref. [26] the $\alpha$ and $\beta$ orbital values for the binding energy and the kinetic energy were averaged. The threshold in the BEB approach depends on the $B$ value for the highest occupied molecular orbital (HOMO) $1 \pi_{g}$, and was obtained subtracting the values for the corresponding orbital in the $\alpha$ and $\beta$ sets. This is the most critical aspect and determines the accuracy of the results. The UHF value of $8.47 \mathrm{eV} \mathrm{[29]} \mathrm{is} \mathrm{in} \mathrm{fact} \mathrm{lower} \mathrm{than} \mathrm{the} \mathrm{expected} \mathrm{value} \mathrm{of} 10.47 \mathrm{eV}$, which corresponds to the first allowed, one-electron process of ionization connecting the metastable state of the $\mathrm{N}_{2}$ molecule to the first excited state of the molecular ion, i.e., $\mathrm{N}_{2}\left(A^{3} \Sigma_{u}^{+}\left(1 \pi_{u}^{3} 3 \sigma_{g}^{2} 1 \pi_{g}^{1}\right)\right)-e\left(1 \pi_{g}\right) \rightarrow \mathrm{N}_{2}^{+}\left(A^{2} \Pi_{u}\left(1 \pi_{u}^{3} 3 \sigma_{g}^{2}\right)\right)$. This process is highly favored 
with respect to the transition to the ground state of $\mathrm{N}_{2}^{+}\left(X^{2} \Sigma_{g}^{+}\left(1 \pi_{u}^{4} 3 \sigma_{g}^{1}\right)\right)$, with a threshold of $9.41 \mathrm{eV}$, that would require a two electron transition.

The total ionization cross section is displayed in Figure 1, also plotting the results obtained artificially modifying the binding energy of the $1 \pi_{g}$ orbital, $B^{H O M O}$, to reproduce the experimental ionization threshold, as suggested in Ref. [26], the cross section being shifted in energy and slightly lowered in its maximum value. It is interesting to compare the BEB cross sections with the results obtained in the framework of the Complex Scattering Potential-ionization contribution (CSP-ic) formalism [23]. Actually, in this theoretical treatment the ionization threshold is an external parameter and two model were proposed, model A based on the theoretical value for the formation of the $\mathrm{N}_{2}^{+}\left(X^{2} \Sigma_{g}^{+}\right)$state, $9.41 \mathrm{eV}$, and model $\mathrm{B}$ where the value $10.1 \mathrm{eV}$, corresponding to the appearance potential in Ref. [33], is chosen. The two methods agree quite well and both predict an ionization cross section that, regardless the threshold, is greater than the experiments, also reported in Figure 1. In fact, the $\mathrm{N}_{2}\left(A^{3} \Sigma_{u}^{+}\right)$state is the only molecular metastable state investigated experimentally $[33,34]$, the molecular beam prepared by quasi-resonant asymmetric charge transfer neutralization and with subsequent ionization by electron beam. The two measures were done with a different charge-transfer gas and the existence of the metastable in the neutralized beam was postulated on the basis of the observed lowering of the ionization threshold with respect to the ground state. As mentioned the apparent threshold in Ref. [33] is lower than the theoretical value predicted for the $\mathrm{N}_{2}(A) \rightarrow \mathrm{N}_{2}^{+}(A)$ process and it was attributed to presence of vibrationally excited $\mathrm{N}_{2}(A)$ molecules in the beam. The reasons for discrepancies between theory and experiments, could be attributed to the fact that all channels are accounted for in the total ionization cross section, while experiments focus on the nondissociative ionization process. Furthermore, the procedure for the separation of ground and metastable contributions to the ion signal is critical and could be a source of uncertainty in the measure.

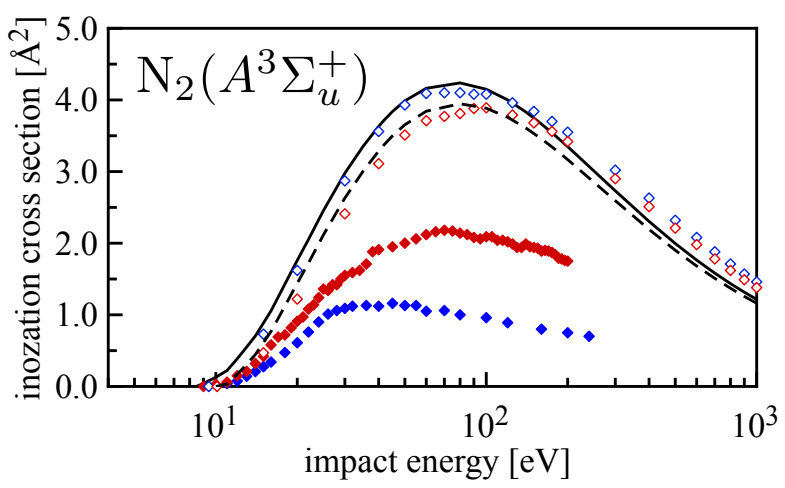

Figure 1. BEB cross sections for total ionization (solid lines) of $\mathrm{N}_{2}\left(A^{3} \Sigma_{u}^{+}\right)$metastable [29], (dashed line) $\mathrm{BEB}$ cross section with $B^{H O M O}=10.47 \mathrm{eV}$. CSP-ic results [23] for model A (blue open diamonds) and model B (red open diamonds). Experiments: (close blue diamonds) [33], (close red diamonds) [34].

The total ionization for the metastable state of $\mathrm{CO}$ molecule (Figure 2) was derived in the BEB approach [29], performing multiconfiguration self-consistent field (MCSCF) calculations not only at $R_{e q}=1.20574 \AA$, but also varying the molecular geometry, confirming the dominant role of the configuration $\left(3 \sigma^{2} 4 \sigma^{2} 5 \sigma 1 \pi^{4} 2 \pi\right)$. The orbital parameters were again obtained with unrestricted Hartree Fock approach, finding the ionization threshold at $9.50 \mathrm{eV}$. This value is greater than the one estimated from the energy spectrum, $7.97 \mathrm{eV}$, corresponding to the one-electron ionization $\mathrm{CO}\left(a^{3} \Pi\right)-e(2 \pi) \rightarrow \mathrm{CO}^{+}\left({ }^{2} \Sigma^{+}\right)$as lowest-threshold channel. 


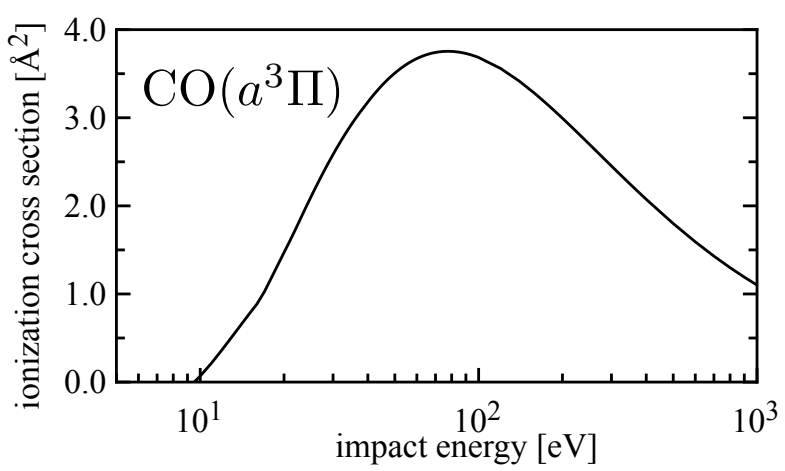

Figure 2. BEB cross sections for total ionization of $\mathrm{CO}\left(a^{3} \Pi\right)$ metastable [29].

In the case of $\mathrm{H}_{2}$, the ionization cross sections of the metastable $c^{3} \Pi_{u}$, of the other bound triplet $a^{3} \Sigma_{g}^{+}$and of the three lowest singlet excited states were calculated firstly with the classical Gryzinski approach [35], deriving vibrationally-resolved datasets relevant to collisional-radiative models, and recently re-evaluated with the accurate MCCC approach [24] for the fundamental vibrational level $v=0$ of the excited states. The total ionization for the $c$ state was also estimated within the complex potential (CSP-ic) formalism [36], considering two models based on a different choice of the lowest threshold energy for the ionization. In model A, the energy limit was set to $2.82 \mathrm{eV}$, derived from the theoretical energy of the excited $c^{3} \Pi_{u}$ state estimated within the R-matrix approach [37], while in model $\mathrm{B}$, the ionization was fixed at the experimental value $3.66 \mathrm{eV}$ [38]. The significance of this choice is evident in the threshold behavior of the cross section, affecting the low-temperature rate of the process, however at high collision energies the two models converge.

Here, the two triplets $c^{3} \Pi_{u}$ and $a^{3} \Sigma_{g}^{+}$are considered. In the ab initio step, MCSCF electronic structure calculations were preliminary performed, with the aug-cc-pV5Z basis set, at the equilibrium internuclear distance of each electronic state, confirming that a single determinant representation is accurate, being the configuration coefficient close to unity for both states. The excited configurations, $\left(1 \sigma_{g} 1 \pi_{u}\right)$ for $\left(c^{3} \Pi_{u}\right)$ and $\left(1 \sigma_{g} 2 \sigma_{g}\right)$ for $a^{3} \Sigma_{g}^{+}$, were then treated in the EKT (extended Koopmans' theorem) method [39], available in the GAMESS code, obtaining a quite accurate estimation of the ionization potential values. In fact, in the case of the $a$ state the binding energy of the excited orbital, $3.6245 \mathrm{eV}$, is very close to the experimental threshold value $3.639 \mathrm{eV}$, while for the $c$ state the EKT value, $3.31702 \mathrm{eV}$, is lower than the accurate threshold at $3.66 \mathrm{eV}$. The orbital parameters for the two states are reported in Table 1.

Table 1. Orbital binding energy, occupation number, orbital symmetry, and kinetic energy for $\mathrm{H}_{2}\left(c^{3} \Pi_{u}\right)\left(1 \sigma_{g} 1 \pi_{u}\right)$ and $\mathrm{H}_{2}\left(a^{3} \Sigma_{g}^{+}\left(1 \sigma_{g} 2 \sigma_{g}\right)\right.$ states at the corresponding equilibrium internuclear distance, i.e., $R_{e q}^{c}=1.0376 \AA$ and $R_{e q}^{a}=0.98879 \AA$.

\begin{tabular}{ccccc}
\hline & $\boldsymbol{B}[\mathbf{e V}]$ & $\boldsymbol{N}$ & & $\boldsymbol{U}[\mathbf{e V}]$ \\
\hline \multirow{2}{*}{$c^{3} \Pi_{u}$} & 22.59329 & 1 & $\mathrm{Ag}$ & 15.53490 \\
& 3.31702 & 1 & $\mathrm{~B} 2 \mathrm{u}$ & 5.66877 \\
\hline \multirow{2}{*}{$a^{3} \Sigma_{g}^{+}$} & 25.5348 & 1 & $\mathrm{Ag}$ & 17.2390 \\
& 3.6245 & 1 & $\mathrm{Ag}$ & 2.7310 \\
\hline
\end{tabular}

In Figure 3, the total ionization cross sections for the $c^{3} \Pi_{u}$ and $a^{3} \Sigma_{g}^{+}$states of $\mathrm{H}_{2}$ are displayed as a function of collision energy and compared with the other theoretical results. For the metastable, the Gryzinski and CSP-ic approaches both give values in good agreement with the quantum MCCC cross section, this last representing the reference, while the BEB model is about $10 \%$ lower at the maximum, the error reducing at higher energies. Differently, for the $a$ state the BEB cross section compares significantly better with the accurate MCCC values, where the Gryzinski approach overestimates the maximum. 

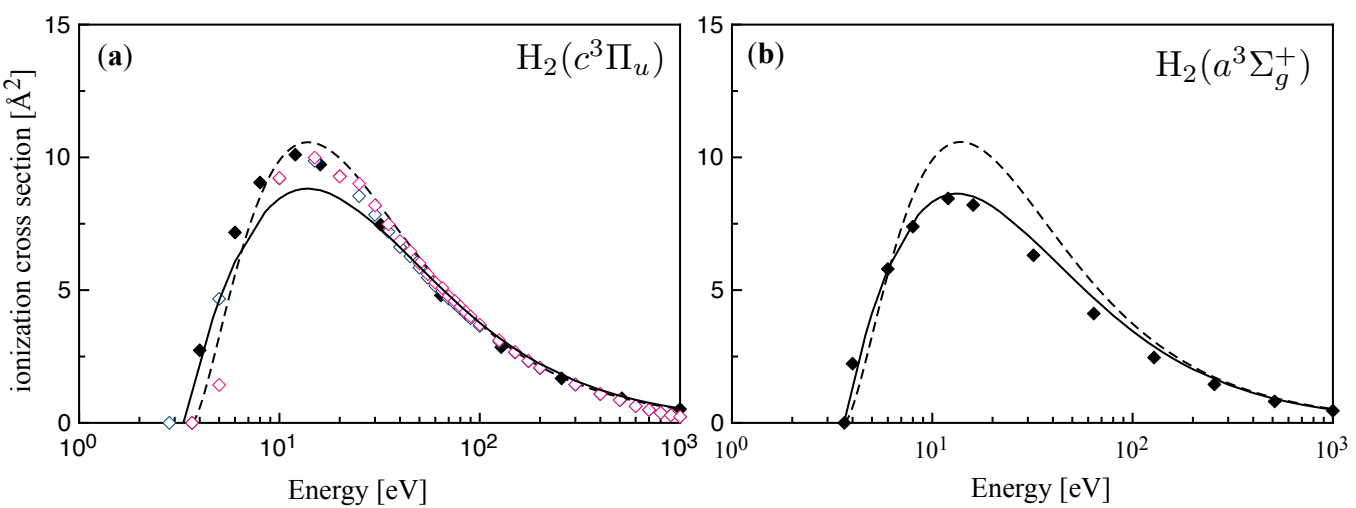

Figure 3. Total ionization cross section of $\mathrm{H}_{2}$ (a) $c^{3} \Pi_{u}$ metastable and (b) $a^{3} \Sigma_{g}^{+}$states. (solid line) BEB model; (dashed line) classical Gryzinski approach [14]; (close diamonds) MCCC method [24,40]; CSP-ic method [36] models A (blue open diamonds) and B (red open diamonds).

The ionization from excited states is characterized not only by the lowering of the energy threshold for the process, but also by a significant enhancement of the absolute value of the cross section with respect to the ground state. In fact, the peak value increases of a factor from 1.5 for $\mathrm{N}_{2}$ and 1.7 for $\mathrm{CO}$ to 10 for $\mathrm{H}_{2}$. In the hydrogen case, the enhancement factor is that large because of the significant difference in the binding energy of the ejected electron in the excited configurations with respect to the ground closed-shell configuration, that, in turn, favors the ionization process. The BEB model accuracy is acceptable also in the case of excited metastable states and related to the accuracy of the ab initio biding energy of the highest occupied orbital.

\section{Conclusions}

The derivation of cross sections for electron-impact-induced processes in metastable states of molecular species is a requirement for the creation of a complete kinetic scheme of nonequilibrium technological plasmas. Among electron-scattering processes, the ionization is key in the onset of electron density and the reduction of the threshold energy, when initiated from excited states, largely enhances the effect. The BEB model for ionization was widely used in the literature to estimate the cross sections for atoms and molecules in their ground states, due to the noticeable accuracy of results despite the simplicity of the formulation, free of external parameters, being the orbital values entering the working equation obtainable by standard electronic structure calculations. In this paper, the total ionization cross sections for metastables of diatomic molecules estimated within the framework of the BEB approach are discussed, comparing the results with those obtained with other more sophisticated theoretical methods, confirming discrepancies within $10 \%$ also characterizing the ground state calculations.

Author Contributions: Conceptualization and software, A.L.; analysis and discussion, A.L., R.C. and G.C.; writing-original draft preparation, A.L.; writing-review, A.L., R.C. and G.C. All authors have read and agreed to the published version of the manuscript.

Funding: This research received no external funding.

Conflicts of Interest: The authors declare no conflict of interest.

\section{References}

1. Pietanza, L.D.; Guaitella, O.; Aquilanti, V.; Armenise, I Bogaerts, A.; Capitelli, M.; Colonna, G.; Guerra, V.; Engeln, R.; Kustova, E.; Lombardi, A.; et al. Advances in non-equilibrium $\mathrm{CO}_{2}$ plasma kinetics: A theoretical and experimental review. Eur. Phys. J. D 2021, 75, 237. [CrossRef]

2. Starikovskaia, S.; Lacoste, D.A.; Colonna, G. Non-equilibrium plasma for ignition and combustion enhancement. Eur. Phys. J. D 2021, 75, 231. [CrossRef]

3. Šimek, M.; Homola, T. Plasma-assisted agriculture: history, presence, and prospects-A review. Eur. Phys. J. D 2021, 75, 210. [CrossRef] 
4. $\quad$ Fridman, A.A.; Friedman, G.G. Plasma Medicine; John Wiley \& Sons: Chichester, UK, 2013.

5. Colonna, G.; Pintassilgo, C.D.; Pegoraro, F.; Cristofolini, A.; Popoli, A.; Neretti, G.; Gicquel, A.; Duigou, O.; Bieber, T.; Hassouni, K.; et al. Theoretical and experimental aspects of non-equilibrium plasmas in different regimes: Fundamentals and selected applications. Eur. Phys. J. D 2021, 75, 183. [CrossRef]

6. Capitelli, M.; Celiberto, R.; Colonna, G.; Esposito, F.; Gorse, C.; Hassouni, K.; Laricchiuta, A.; Longo, S. Fundamental Aspects of Plasma Chemical Physics: Kinetics; Springer Series on Atomic, Optical, and Plasma Physics; Springer: New York, NY, USA, 2016; Volume 85.

7. Colonna, G. On the relevance of superelastic collisions in argon and nitrogen discharges. Plasma Sources Sci. Technol. 2020, 29, 065008. [CrossRef]

8. Ricard, A.; Oh, S.G.; Guerra, V. Line-ratio determination of atomic oxygen and $\mathrm{N}_{2}\left(A^{3} \Sigma_{u}^{+}\right)$metastable absolute densities in an RF nitrogen late afterglow. Plasma Sources Sci. Technol. 2013, 22, 035009. [CrossRef]

9. Colonna, G.; Capitelli, M. The influence of atomic and molecular metastable states in high-enthalpy nozzle expansion nitrogen flows. J. Phys. D Appl. Phys. 2001, 34, 1812. [CrossRef]

10. Pietanza, L.D.; Colonna, G.; Capitelli, M. Non-equilibrium plasma kinetics of reacting CO: An improved state to state approach. Plasma Sources Sci. Technol. 2017, 26, 125007. [CrossRef]

11. Starik, A.M.; Kozlov, V.E.; Titova, N.S. On the influence of singlet oxygen molecules on the speed of flame propagation in methane-air mixture. Combust. Flame 2010, 157, 313-327. [CrossRef]

12. Wünderlich, D.; Scarlett, L.H.; Briefi, S.; Fantz, U.; Zammit, M.C.; Fursa, D.V.; Bray, I. Application of molecular convergent close-coupling cross sections in a collisional radiative model for the triplet system of molecular hydrogen. J. Phys. D Appl. Phys. 2021, 54, 115201. [CrossRef]

13. Celiberto, R.; Armenise, I.; Cacciatore, M.; Capitelli, M.; Esposito, F.; Gamallo, P.; Janev, R.; Laganà, A.; Laporta, V.; Laricchiuta, A.; et al. Atomic and molecular data for spacecraft re-entry plasmas. Plasma Sources Sci. Technol. 2016, 25, 033004. [CrossRef]

14. Wünderlich, D. Vibrationally resolved ionization cross sections for the ground state and electronically excited states of the hydrogen molecule and its isotopomeres. At. Data Nucl. Data Tables 2021, 140, 101424. [CrossRef]

15. Celiberto, R.; Janev, R.K.; Laricchiuta, A.; Capitelli, M.; Wadehra, J.M.; Atems, D.E. Cross section data for electron-impact inelastic processes of vibrationally excited molecules of hydrogen and its isotopes. At. Data Nucl. Data Tables 2001, 77, 161-213. [CrossRef]

16. Celiberto, R.; Capitelli, M.; Cacciatore, M. Electron impact direct dissociative-ionization cross sections from vibrationally excited $\mathrm{H}_{2}$ molecules and translational energy distribution functions of protons. Chem. Phys. 1990, 140, 209-215. [CrossRef]

17. Cacciatore, M.; Capitelli, M.; Gorse, C. Non-equilibrium dissociation and ionization of nitrogen in electrical discharges: the role of electronic collisions from vibrationally excited molecules. Chem. Phys. 1982, 66, 141-151. [CrossRef]

18. Bauer, E.; Bartky, C.D. Calculation of inelastic electron-molecule collision cross sections by classical methods. J. Chem. Phys. 1965, 43, 2466-2476. [CrossRef]

19. Gryziński, M. Classical Theory of Atomic Collisions. I. Theory of Inelastic Collisions. Phys. Rev. 1965, 138, A336-A358, doi:10.1103/PhysRev.138.A336. [CrossRef]

20. Kosarim, A.V.; Smirnov, B.M.; Capitelli, M.; Celiberto, R.; Petrella, G.; Laricchiuta, A. Ionization of excited nitrogen molecules by electron impact. Chem. Phys. Lett. 2005, 414, 215-221. doi:10.1016/j.cplett.2005.08.012. [CrossRef]

21. Kosarim, A.V.; Smirnov, B.M.; Capitelli, M.; Laricchiuta, A.; Paniccia, F. Electron impact ionization cross sections of vibrationally and electronically excited oxygen molecules. Chem. Phys. Lett. 2006, 422, 513-517. [CrossRef]

22. Laricchiuta, A.; Capitelli, M.; Celiberto, R.; Colonna, G. Dissociation and Ionization cross Sections and Rate Coefficients of Air Molecules by Electron Impact: The Role of Vibrational Energy; AIAA Paper 2006-2898; American Institute of Aeronautics and Astronautics: Reston, VA, USA, 2006.

23. Joshipura, K.N.; Gangopadhyay, S.S.; Kothari, H.N.; Shelat, F.A. Total electron scattering and ionization of N, $\mathrm{N}_{2}$ and metastable excited $\mathrm{N}_{2}^{\star}\left(\mathrm{A}^{3} \Sigma_{u}^{+}\right)$: Theoretical cross sections. Phys. Lett. A 2009, 373, 2876-2881. [CrossRef]

24. Scarlett, L.H.; Savage, J.S.; Fursa, D.V.; Bray, I.; Zammit, M.C.; Schneider, B.I. Convergent close-coupling calculations of electrons scattering on electronically excited molecular hydrogen. Phys. Rev. A 2021, 103, 032802. [CrossRef]

25. Kim, Y.K.; Rudd, M.E. Binary-encounter-dipole model for electron-impact ionization. Phys. Rev. A 1994, 50, 3954. [CrossRef] [PubMed]

26. Hwang, W.; Kim, Y.K.; Rudd, M.E. New model for electron-impact ionization cross sections of molecules. J. Chem. Phys. 1996, 104, 2956-2966. [CrossRef]

27. Huber, S.E.; Mauracher, A.; Süß, D.; Sukuba, I.; Urban, J.; Borodin, D.; Probst, M. Total and partial electron impact ionization cross sections of fusion-relevant diatomic molecules. J. Chem. Phys. 2019, 150, 024306. [CrossRef] [PubMed]

28. Kim, Y.K.; Desclaux, J.P. Ionization of carbon, nitrogen, and oxygen by electron impact. Phys. Rev. A 2002, 66, 012708. [CrossRef]

29. Laricchiuta, A.; Pietanza, L.D.; Capitelli, M.; Colonna, G. Electron-CO excitation and ionization cross sections for plasma modeling. Plasma Phys. Control. Fusion 2018, 61, 014009. [CrossRef]

30. Tanaka, H.; Brunger, M.J.; Campbell, L.; Kato, H.; Hoshino, M.; Rau, A.R.P. Scaled plane-wave Born cross sections for atoms and molecules. Rev. Mod. Phys. 2016, 88, 025004. [CrossRef]

31. Schmidt, M.W.; Baldridge, K.K.; Boatz, J.A.; Elbert, S.T.; Gordon, M.S.; Jensen, J.H.; Koseki, S.; Matsunaga, N.; Nguyen, K.A.; $\mathrm{Su}, \mathrm{S}$; ; et al. General atomic and molecular electronic structure system. J. Comput. Chem. 1993, 14, 1347-1363. [CrossRef]

32. Gordon, M.S.; Schmidt, M.W. Theory and Applications of Computational Chemistry: The First Forty Years; Dykstra, C.E., Frenking, G., Kim, K.S., Scuseria, G.E., Eds.; Elsevier: Amsterdam, The Netherlands, 2011; pp. 1167-1189. 
33. Armentrout, P.B.; Tarr, S.M.; Dori, A.; Freund, R.S. Electron impact ionization cross section of metastable $\mathrm{N}_{2}\left(\mathrm{~A}^{3} \Sigma_{u}^{+}\right)$. J. Chem. Phys. 1981, 75, 2786-2794. [CrossRef]

34. Freund, R.S.; Wetzel, R.C.; Shul, R.J. Measurements of electron-impact-ionization cross sections of $\mathrm{N}_{2}, \mathrm{CO}_{2} \mathrm{CO}_{2}, \mathrm{CS}_{2} \mathrm{~S}_{2}, \mathrm{CS}_{2}$, and metastable $\mathrm{N}_{2}$. Phys. Rev. A 1990, 41, 5861. [CrossRef] [PubMed]

35. Wünderlich, D. Vibrationally resolved ionization cross sections for the ground state and electronically excited states of the hydrogen molecule. Chem. Phys. 2011, 390, 75-82. [CrossRef]

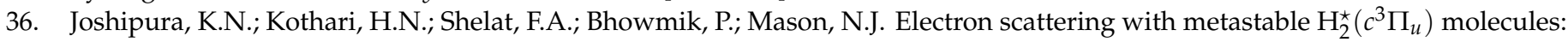
ionization and other total cross sections. J. Phys. At. Mol. Opt. Phys. 2010, 43, 135207. [CrossRef]

37. Branchett, S.E.; Tennyson, J.; Morgan, L.A. Electronic excitation of molecular hydrogen using the r-matrix method. J. Phys. B At. Mol. Opt. Phys. 1990, 23, 4625. [CrossRef]

38. Herzberg, G. Molecular Spectra and Molecular Structure. 1. Spectra of Diatomic Molecules; Van Nostrand: New York, NY, USA, 1950.

39. Morrison, R.C. The extended Koopmans' theorem and its exactness. J. Chem. Phys. 1992, 96, 3718-3722. [CrossRef]

40. MCCC. Molecular Convergent Close-Coupling Database. 2021. Available Online: https://www.mccc-db.org/ (accessed on 19 December 2021). 